\title{
Cut-off features in interplanetary solar radio type IV emission
}

\author{
Silja Pohjolainen* \\ Tuorla Observatory, Department of Physics and Astronomy, University of Turku, 20014 TURUN YLIOPISTO, Finland
}

Nasrin Talebpour Sheshvan

Department of Physics and Astronomy, University of Turku, 20014 TURUN YLIOPISTO, Finland

\begin{abstract}
Solar radio type IV bursts can sometimes show directivity, so that no burst is observed when the source region in located far from the solar disk center. This has recently been verified also from space observations, at decameter wavelengths, using a 3D-view to the Sun with STEREO and Wind satellites. It is unclear whether the directivity is caused by the emission mechanism, by reduced radio wave formation toward certain directions, or by absorption/blocking of radio waves along the line of sight. We present here observations of three type IV burst events that occurred on 23, 25, and 29 July 2004, and originated from the same active region. The source location of the first event was near the solar disk center and in the third event near the west limb. Our analysis shows that in the last two events the type IV bursts experienced partial cut-offs in their emission, that coincided with the appearance of shock-related type II bursts. The type II bursts were formed at the flanks and leading fronts of propagating coronal mass ejections (CMEs). These events support the suggestion of absorption toward directions where the type II shock regions are located.
\end{abstract}

Keywords: Sun; Radio emission; Flare; Coronal mass ejection; CME; Space weather

\section{Introduction}

Flares and coronal mass ejections (CMEs) are solar transients that can accelerate particles to high energies and create radio emission within large wavelength ranges, see reviews in Pick and Vilmer (2008) and Nindos et al. (2008). At low frequencies, below $1 \mathrm{GHz}$, the radio emission is most often plasma emission as the trapped or propagating particles cause oscillations in the surrounding medium and create radio waves that appear at the local plasma frequency and/or its harmonics. The emission frequency can then be used as a direct measure of density, and an indirect measure of height, in the solar atmosphere (White, 2007). Gyrosynchrotron emission is also possible when strong magnetic fields are present, but to be observable the gyrofrequency must then exceed the local plasma frequency (Dulk, 1985).

Solar radio type IV bursts were studied intensively in the 1960s and their main features are described in Kundu (1965). The main division was made between moving and stationary bursts: the former are moving in solar altitude and the latter are located low in the corona with no systematic movement (Boischot, 1957). Moving type IVs were estimated to be non-directive and emitting mainly weakly-polarized synchrotron emission, while stationary

\footnotetext{
*Corresponding author

Email addresses: silpoh@utu.fi (Silja Pohjolainen), natash@utu.fi (Nasrin Talebpour Sheshvan)
}

bursts were highly directive toward the center of the solar disk and emitted strongly-polarized plasma radiation. The cause for stationary type IV bursts could be flare loops, located high in the corona and filled with non-thermal particles. Moving type IV bursts would require a propagating CME or an expanding arch, to create a frequency-drifting structure in the dynamic spectra.

The fine structures of metric type IV bursts, typically embedded in burst continua, can be used to trace the magnetic field restructuring and the corresponding energy release in the low corona (Bouratzis et al., 2015). The observed fine structures include type III-like short duration bursts, zebra patterns, and stripes with both positive and negative frequency drifts (Melnik et al., 2008; Bouratzis et al., 2016).

Before space instruments the observable frequency range for type IV bursts was limited to meter-decameter waves by the Earth atmosphere, but since the early 1990s radio dynamic spectra have been available from decameterhectometer $(\mathrm{DH})$ to $\mathrm{km}$ waves. Observations at $\mathrm{DH}$ wavelengths probe the plasma conditions and transients in the interplanetary (IP) space, and currently radio spectra are available from the spectrographs on board Wind (Bougeret et al., 1995) and STEREO (Bougeret et al., 2008) satellites.

IP type IV bursts observed by the Wind/WAVES in 1998-2012 have been listed by Hillaris, Bouratzis, and Nindos (2016). They determined the bursts to be moving type 
Table 1: Flares and CMEs associated with radio type IV bursts.

\begin{tabular}{|c|c|c|c|c|c|c|c|c|}
\hline ууyy-mm-dd & $\begin{array}{l}\text { Flare } \\
\text { start } \\
\text { (UT) }\end{array}$ & $\begin{array}{l}\text { GOES } \\
\text { class }\end{array}$ & $\begin{array}{l}\text { AR } \\
\text { location }\end{array}$ & $\begin{array}{c}\text { Metric type IV } \\
\text { burst start } \\
\text { (UT) }\end{array}$ & $\begin{array}{l}\text { IP type IV } \\
\text { burst } 1 \\
\text { (UT) }\end{array}$ & $\begin{array}{l}\text { IP type II } \\
\text { burst } \\
\text { (UT) }\end{array}$ & $\begin{array}{c}\mathrm{CME} \\
\text { height }^{2} \\
\left(\mathrm{R}_{\odot}\right)\end{array}$ & $\begin{array}{l}\text { CME } \\
\text { speed }^{3} \\
\left(\mathrm{~km} \mathrm{~s}^{-1}\right)\end{array}$ \\
\hline \multirow[t]{2}{*}{ 2004-07-23 } & $17: 07$ & M2.2 & N03W04 & $19: 10$ & $19: 30-21: 00$ & $18: 30-19: 30$ & 8.0 & CME1: 600 \\
\hline & 18:02 & $\mathrm{C} 4.1$ & N05W05 & & & & 4.5 & CME2: 550 \\
\hline \multirow[t]{2}{*}{ 2004-07-25 } & $13: 37$ & M2.2 & N04W30 & $14: 20$ & $15: 10-20: 00$ & $15: 30-19: 00$ & 4.7 & CME1: 450 \\
\hline & $14: 19$ & M1.1 & N08W33 & & & $17: 40-$ & 4.3 & CME2: 1300 \\
\hline 2004-07-29 & $11: 42$ & $\mathrm{C} 2.1$ & W-limb & $11: 20$ & $12: 40-14: 30$ & $13: 30-$ & 4.5 & 1000 \\
\hline
\end{tabular}

${ }^{1}$ Start time defined as appearance at $14 \mathrm{MHz}$ (Wind/WAVES upper frequency limit) .

${ }^{2} \mathrm{CME}$ leading front height at the time of IP type IV appearance (from fits to the height data if not observed).

${ }^{3} \mathrm{CME}$ speed at the time of IP type IV appearance.

IVs on the basis that their emission drifted to DH wavelengths. However, as there is no radio imaging available at $\mathrm{DH}$ waves at present, their actual movement in altitude is difficult to verify.

The directivity in IP type IV bursts was first reported by Gopalswamy et al. (2016). By comparing the radio spectra observed from three different viewing angles around the Sun (STEREO A and B, and Wind), they observed that the IP type IV bursts are visible only if their source location is within $\sim 60$ degrees from the Sun center. Their conclusion was that the type IV emission is directed along a narrow cone above the flare site, the emission mechanism is most probably plasma emission, and the type IV bursts are stationary, i.e., not associated with moving CME structures. However, in a later case study Melnik et al. (2018) were able to show that the IP type IV burst that was not observed by Wind near Earth (but visible in both STEREO A and B dynamic spectra), could still be observed from Earth at decameter waves, by URAN-2.

Recently, Talebpour Sheshvan and Pohjolainen (2018), who used similar data from the three spacecraft, suggested that the observed directivity of IP type IV bursts may not be due to the emission directivity, but could be caused by absorption or suppression of emission toward certain viewing angles. The absorber, i.e., dense plasma along the line-of-sight, would block the emission at longer DH wavelengths. Such high-density regions could be formed by shock-streamer interaction, especially near CME flanks.

This article presents the analysis of three separate eruption events that occurred in July 2004 (before STEREO was launched and therefore only Earth view available). These events all included flares, CMEs, type II bursts, and metric and DH type IV bursts. They originated from the same active region, which rotated from a location near the center of the disk (first event) to the west limb (third event), and thus made it possible to observe the type IV bursts and the associated features near the solar disk center and near the limb.

In the analysis we used radio spectral data from Wind satellite, Radio Solar Telescope Network (RSTN) stations, Green Bank Solar Radio Burst Spectrometer (GBSRBS), and Nancay Decameter Array (NDA). Nancay Radiohelio- graph $(\mathrm{NRH})$ images were used to locate the burst sources, but these observations were available only for the two European daytime events. SOHO/LASCO coronagraph data and $\mathrm{SOHO} / \mathrm{EIT}$ images in EUV were taken from the CDAW CME Catalog at https://cdaw.gsfc.nasa.gov/.

\section{Data analysis: solar events in July 2004}

In July 2004 the NOAA solar Active Region (AR) 10652 produced three eruption events that were associated with flares, CMEs, and radio type II and type IV bursts. The events occurred on 23 July (eruption source location at N05W05), 25 July (source location at N04W30), and 29 July (source at west limb). The flares had moderate intensities, with GOES X-ray classes ranging from C2.1 to M2.2. The CMEs were observed in projection, which may affect their height estimates especially when the CME is propagating toward the observer. Fits to the height estimates gave CME speeds that ranged from $450 \mathrm{~km} \mathrm{~s}^{-1}$ to $1300 \mathrm{~km} \mathrm{~s}^{-1}$, see Table 1 .

In all three events both a metric type IV burst (groundbased observations at frequencies higher than $20 \mathrm{MHz}$ ) and an IP type IV burst (space observations at frequencies lower than $14 \mathrm{MHz}$ ) were observed, and they look to be related, i.e., the IP emission was a continuation of the metric emission.

The heights of plasma emission sources can be estimated using solar atmospheric density models, as the frequency of plasma emission depends only on the local plasma density, see, e.g., Pohjolainen et al. (2007). The 'hybrid' atmospheric density model of Vrsnak, Magdalenic, and Zlobec (2004) was selected for our analysis because it was developed to merge the high-density low corona models to the low-density IP models without breaks or discrepancies, which fits the purpose of our analysis.

\subsection{Event on 23 July 2004}

The event originated near the disk center, at $\sim$ N05W05, and was associated with continuous flaring from AR 10652 (see Table 1). Two CMEs were launched from this region within the time period of 17-19 UT, the first one (CME1) was a partial halo-type and the second one (CME2) had 

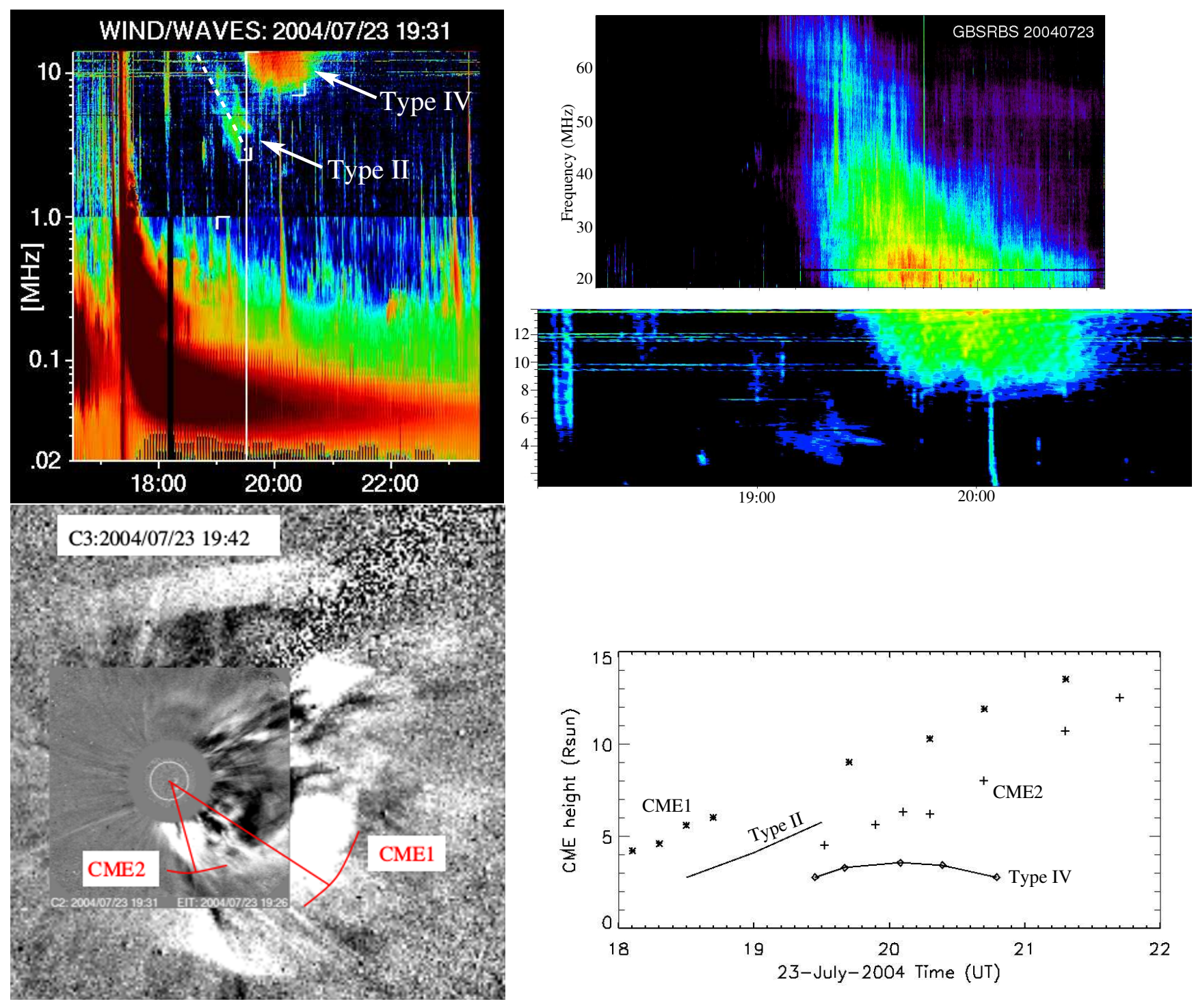

Figure 1: Solar event on 23 July 2004, with source location at N05W05. The type II burst is indicated by a dashed line in the Wind/WAVES dynamic spectrum (top left). The metric type IV burst (GBSRBS) continues to DH wavelengths (Wind/WAVES), their dynamic spectra are both plotted in linear scale (top right). The event included two separate CMEs, shown in the combined LASCO C2/C3 difference image near the time of the type II burst disappearance (bottom left). Three streamers were observed on the western side of the Sun, and one of them was located near the southern flank of CME1. The height-time plot (bottom right) shows the estimated type II burst heights (solid line), the estimated type IV burst heights (connected triangles), and the projected CME heights (stars and crosses for the two CMEs).

more narrow width. They were preceded by a halo CME that was last observed at 17:42 UT at a height of $9.5 \mathrm{R}_{\odot}$. It is unclear if the earlier halo CME merged or interacted with CME1, which was first observed at 17:54 UT at height $3.2 \mathrm{R}_{\odot}$.

A metric type IV burst was observed to start at 19:10 UT, observed by GBSRBS and RSTN Palehua stations, and the emission drifted to decameter waves where it was observed by Wind/WAVES. Only one IP type II burst was observed at 18:30-19:30 UT, and its emission ended soon after the appearance of the type IV burst at WAVES frequencies. A faint metric type II burst was observed earlier, at 16:00-16:15 UT, but it was most probably related with the acceleration of the halo-type CME observed before CME1 and CME2.

The height-time plot in Figure 1 shows that the estimated height of the IP type II burst source (the type II lane is indicated with a dashed line in the top left panel) was $2-3 \mathrm{R}_{\odot}$ lower than the (projected) leading front of CME1. The CME2 heights cannot be determined accurately before 19:31 UT, but a filament eruption in the active region is visible in the $\mathrm{SOHO} / \mathrm{EIT}$ images at 18:48 UT. As type II bursts often appear at the time of CME acceleration, it is possible that if the CME2 was launched near 18:30 UT and it was accelerating, it could have been the source of the type II burst. Furthermore, as CME heights are difficult to determine for on-the-disk events, as CMEs are seen as projections, the true CME2 heights could have 

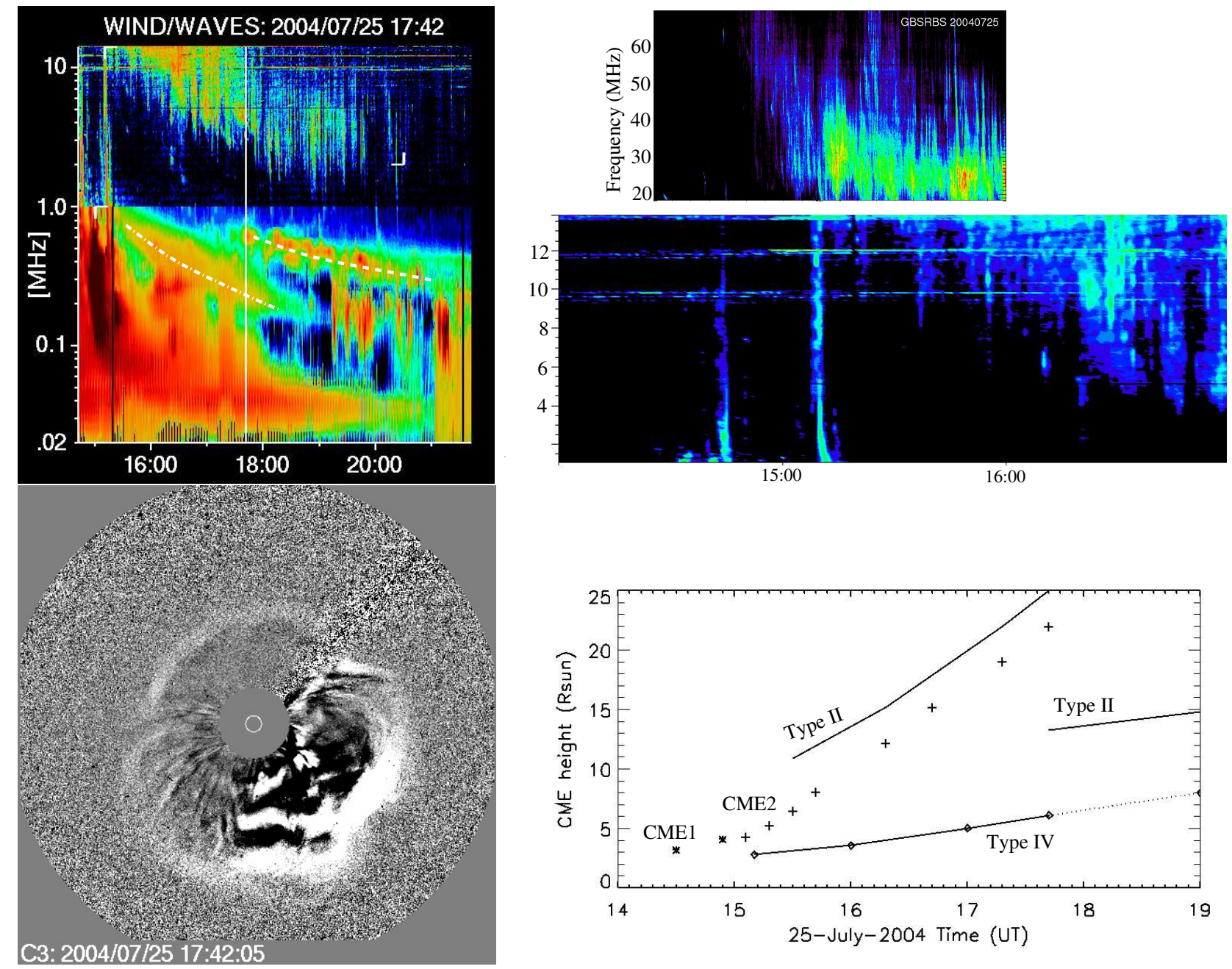

$15: 00$

16:00

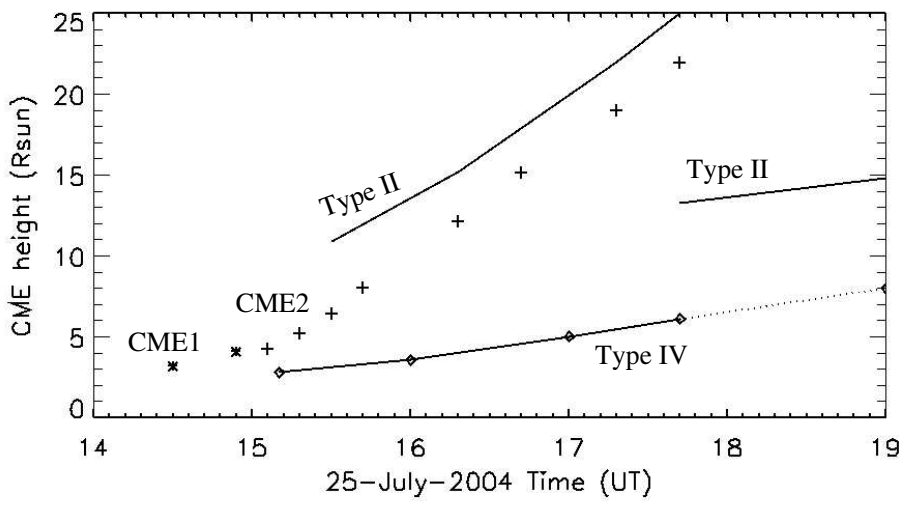

Figure 2: Solar event on 25 July 2004, with source location at N04W30. The first-appearing wide-band type II burst is indicated by a dash-dotted line and the later narrow-band type II burst by a dashed line in the Wind/WAVES dynamic spectrum. The vertical white line indicates the time when the type IV burst intensity started to decrease (top left). The metric type IV burst (GBSRBS) continues to DH wavelengths (Wind/WAVES), their dynamic spectra are plotted in linear scale (top right). The merged CME is shown in the LASCO C3 difference image near the time of the second type II burst appearance and the partial disappearance of the type IV burst (bottom left). Streamers were observed both in the north-west and south-west directions. The height-time plot (bottom right) shows the estimated type II burst heights (solid lines), the estimated type IV burst heights (connected triangles), and the projected CME heights (stars and crosses, the CMEs merge as one near $15 \mathrm{UT})$.

been higher than what was observed. On the other hand, CME1 propagated toward the south-west and a streamer was located at its southern flank, suggesting that a CME flank shock would have been possible, too.

The type II burst disappeared from the spectrum near 19:30 UT, having reached $3 \mathrm{MHz}$ frequency, which corresponds to a source height of about $5.7 \mathrm{R}_{\odot}$. The type II burst source was then $\sim 1 \mathrm{R}_{\odot}$ higher than the leading front of CME2 and $\sim 2 \mathrm{R}_{\odot}$ lower than that of CME1. Near this time both CMEs had relatively low speeds, 550$600 \mathrm{~km} \mathrm{~s}^{-1}$, and the abrupt disappearance of the type II burst lane may simply have been caused by the shock speed falling below the local Alfven speed. Or, as in Kong et al. (2015), the type II emission ended once the CME/shock front passed the white-light streamer tip.

The type IV burst appeared in the Wind/WAVES spec- trum at $14 \mathrm{MHz}$ at about 19:30 UT. Figure 1 shows the estimated type IV burst heights that were calculated from the lowest emission frequencies of the type IV emission (leading emission structure), assuming that the frequency drift corresponds to the decreasing plasma density in the surrounding medium.

\subsection{Event on 25 July 2004}

The long-duration M1.1 flare at N08W33 started after a series of impulsive compact flares that occurred over a period of two hours. The soft X-ray images analyzed by Vrsnak et al. (2010) revealed that the eruption formed a large cusp-shaped loop system in between AR 10652 and AR 10653, which was located south of AR 10652. The launched CME was a full halo-type and had high speed and almost constant velocity, $1300 \mathrm{~km} \mathrm{~s}^{-1}$, after 15 UT. A 
slower CME was observed to precede this CME, possibly merging with it near 15 UT (Table 1 and height-time plot in Figure 2).

A metric type IV burst was observed by GBSRBS, RSTN San Vito station, and NDA, and the emission drifted to decameter waves where it was observed by Wind/WAVES. NDA observations show that the burst was only very weakly left-hand polarized. (The NDA spectra are available at the Radio Monitoring website, http://secchirh.obspm. $\mathrm{fr} /$ ). The type IV burst drifted to the WAVES upper frequency limit, $14 \mathrm{MHz}$, at 15:10 UT.

At 15:30 UT a wide-band type II emission lane appeared at $1 \mathrm{MHz}$ (indicated with a dash-dotted line in Figure 2), and a second, more narrow-band and fragmented type II lane at 17:40 UT at a higher frequency (dashed line in Figure 2). The IP type IV burst showed a clear intensity decrease after 17:40 UT, and the wide-band type II emission ended near 19:00 UT.

The calculated heights for the first, wide-band type II burst suggest that it was due to a CME bow shock, as the radio emission sources were located just above the projected heights of the CME leading front. This burst has been analyzed earlier by Pohjolainen, Allawi, and Valtonen (2013), as part of a large sample of wide-band type II bursts, where the majority were concluded to be CME bow shocks.

The second, more narrow-band type II burst appeared at 17:40 UT at higher frequencies, and had therefore much lower heights than the wide-band type II burst. The calculated speed of the second shock is also lower (see heighttime plot in Figure 2), as it would be if the shock was located at the flanks of the CME instead of the fastermoving CME nose. The partial blocking of the type IV burst emission, which also started at 17:40 UT, suggests a connection to the second shock.

\subsection{Event on 29 July 2004}

On 29 July 2004 AR 10652 had rotated to the west limb. A long-duration X-ray flare was observed, associated with a high-speed (1000 $\left.\mathrm{km} \mathrm{s}^{-1}\right)$ halo-type CME. Liu et al. (2010) report that the halo CME consisted of a transequatorial loop system that connected AR 10652 to AR 10653, and the loop system was mainly oriented in the north-south direction. The GOES C2.1 flare is listed to start at 11:42 UT, but there is strong indication that the flare had started earlier on the far side of the Sun.

A type IV burst was formed at meter waves at 11:20 UT, and the burst envelope consisted of narrow-band type IIIlike bursts and fluctuations. The burst envelope drifted to DH wavelengths where Wind/WAVES observed it (Figure 3) until 14:30 UT, down to $\sim 3 \mathrm{MHz}$. Liu et al. (2010) defined this spectral feature as a drifting pulsating structure (DPS) that would be formed within the current sheet below the CME, see their Figure 2. We note that as DPS's are typically observed at decimeter waves, at much lower heights, this interpretation may be questioned. NDA observations show that the type IV burst was only very weakly left-hand polarized.

Only one wide-band DH type II lane was observed (Figure 3, top left dynamic spectrum, indicated by a dashdotted line) and it appeared more than two hours after the start of the metric type IV emission (see Table 1).

The height-time plot in Figure 3 shows that the type II burst was most likely due to a bow shock at the leading front of the CME, initiated by a change in the CME velocity around 13:30 UT. (Note that in the LASCO CDAW catalog the LASCO C3 image at 13:42 UT has not been included in the fits to the CME height data.) Details of the wide-band type II burst and comparison to the CME height data can also be found in Pohjolainen, Allawi, and Valtonen (2013).

Near the time of the wide-band type II burst appearance, the type IV burst started to fade away in the dynamic spectrum, after which it disappeared gradually.

\subsection{Type IV burst features}

The three metric type IV bursts showed similar fine structures inside a diffuse frequency-drifting emission envelope. The fine structures included type III-like bursts that appeared in groups (Figure 4). A simple Lomb-Scargle periodicity test revealed that some of the burst groups were repeated every $\sim 100 \mathrm{~s}$. As type III bursts are generated by particle beams traveling along magnetic field lines, this indicates continuous particle acceleration during the type IV bursts. Hard X-ray observations were available only for the 23 July event (RHESSI satellite data) and they showed X-ray burst activity during 19:15-19:50 UT, especially in the $6-50 \mathrm{keV}$ energy range, but no one-to-one correspondence to the type III bursts were found.

Both positive and negative frequency drifts were observed in the radio spectral data at metric wavelengths. The typical bandwidths of these features were some tens of $\mathrm{MHz}$, indicating that the particle beam paths were confined to relatively short distances. The Wind/WAVES spectral data did not have enough time resolution to determine if these fine structures existed also at longer wavelengths. Positive and negative frequency drifts suggest that particles were trapped inside large loops (flare or CME), where they moved along the magnetic field lines toward both lower and higher density regions.

We note that the diffuse emission inside the envelope was strongest in intensity in the 23 July type IV burst, moderate in the 25 July type IV burst, and relatively weak in the 29 July type IV burst, see Figure 5.

\subsection{Radio imaging}

The events on 25 and 29 July 2004 were imaged by the Nancay Radioheliograph (NRH) at $164 \mathrm{MHz}$. The NRH images on 25 July (Figure 6), taken during the time period of the metric type IV emission at $164 \mathrm{MHz}$, show that the emission source was first located near the western limb but then moved to the south-east. As it was discovered earlier 

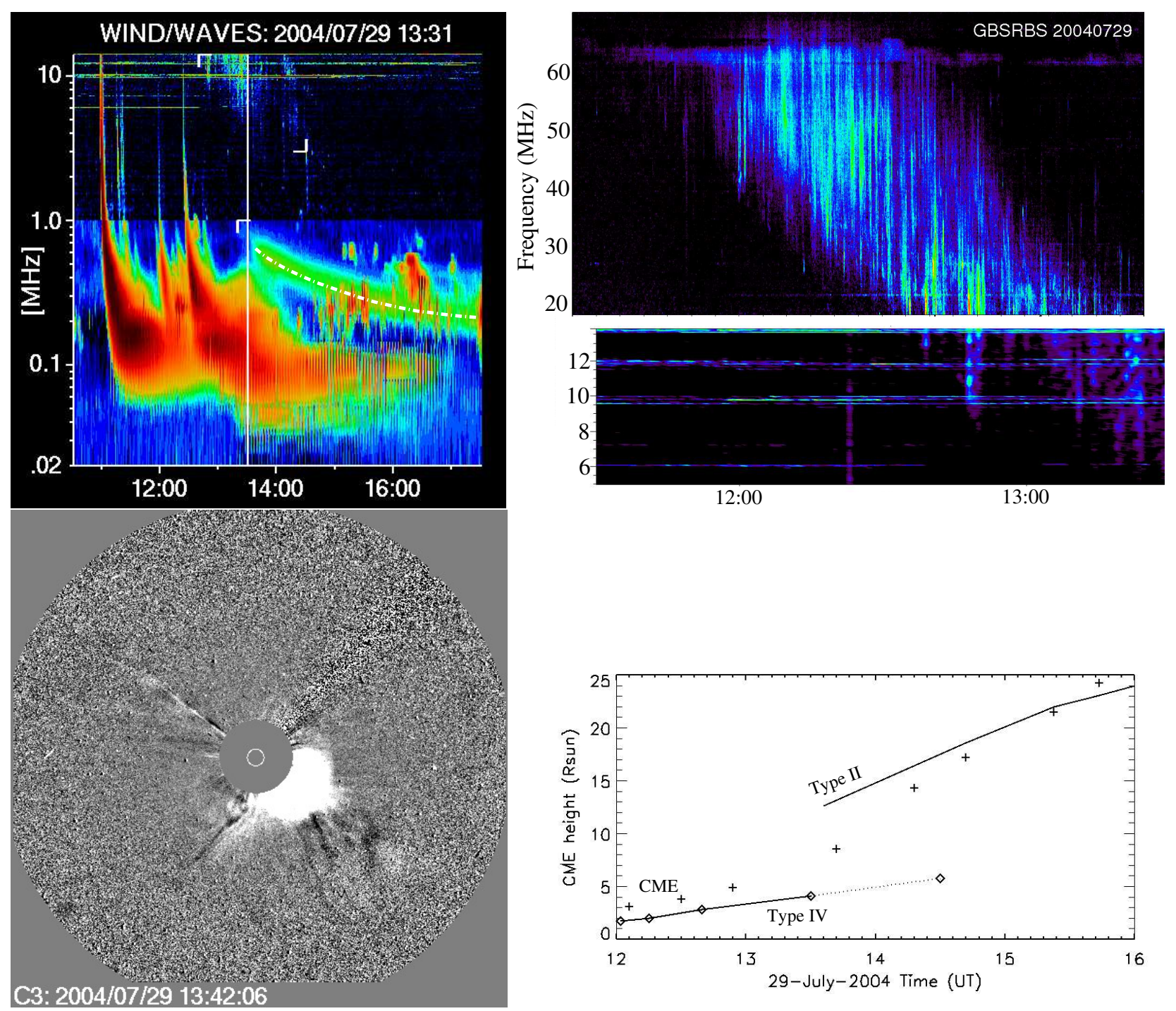

Figure 3: Solar event on 29 July 2004, with source location near the west limb. The wide-band type II burst is indicated by a dash-dotted line in the Wind/WAVES dynamic spectrum. The vertical white line indicates the time when the type IV burst intensity started to decrease (top left). The metric type IV burst (GBSRBS) continues to DH wavelengths (Wind/WAVES), their spectra are plotted in linear scale (top right). The CME is shown in the LASCO C3 difference image near the time of the type II burst appearance and the partial disappearance of the type IV burst (bottom left). Streamers were observed both in the north-west and south-west directions. The height-time plot (bottom right) shows the estimated type II burst heights (solid line), the estimated type IV burst heights (connected triangles), and the projected CME heights (crosses).

(Vrsnak et al., 2010), a large cusp-shaped post-eruption loop system was formed south of AR 10652, connecting it to AR 10653. The radio source movement may therefore have been due to these post-eruption structures located high in the corona, observed projected on the solar disk.

In the 29 July event the metric type IV burst source at $164 \mathrm{MHz}$ was located over the west limb (Figure 7), seemingly near the north-western flank of the CME. We estimated that the heliocentric distance of the emission source was $\sim 1.6 \mathrm{R}_{\odot}$ at $164 \mathrm{MHz}$. Plasma emission at this frequency, in typical atmospheric conditions and densities, is formed near $1.2-1.3 \mathrm{R}_{\odot}$, so the emission may not have been plasma emission.

Imaging observations of a moving type IV burst, using the multifrequency radioheliograph at Clark Lake Radio Observatory, at 125-20 MHz, suggested that a plasmoid containing energetic electrons can result from the disruption of a coronal streamer (Kundu, 1987). Furthermore, Gopalswamy (1990) concluded that as moving type IV bursts primarily depend on non-thermal particles trapped in moving magnetic structures (plasmoids and loops that accompany the CMEs), non-thermal particles can be generated independent of the CME speeds. A particular kind of type IV bursts, expanding CME radio loops, have been imaged by, e.g., Bastian et al. (2001) and Maia et al. (2007). The fast-moving radio loops have been observed to move from a few tenths to more than $1 \mathrm{R}_{\odot}$ above the solar limb. These features have been attributed to inco- 

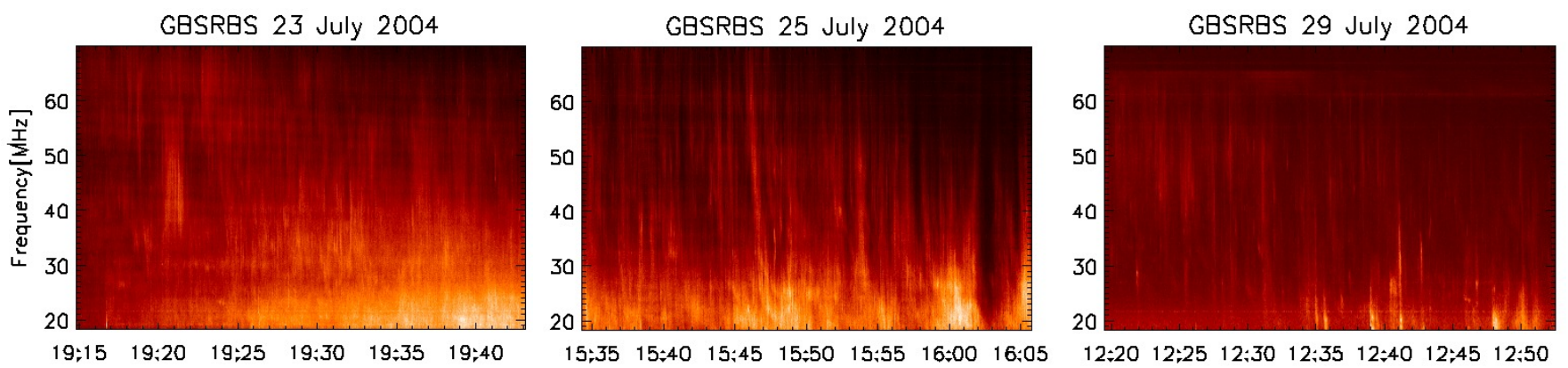

Figure 4: GBSRBS dynamic spectra at 70-20 MHz from the three events show fine structures within the metric type IV continuum emissions.

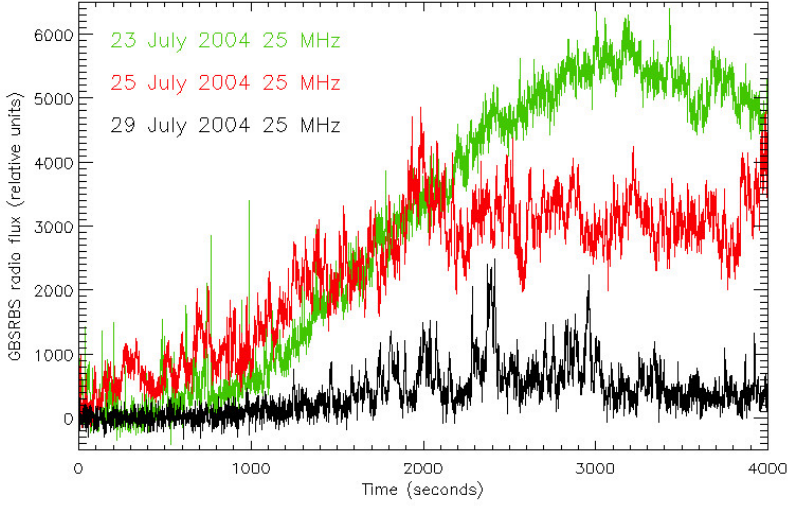

Figure 5: Background subtracted GBSRBS radio flux at $25 \mathrm{MHz}$ in the three type IV burst events.

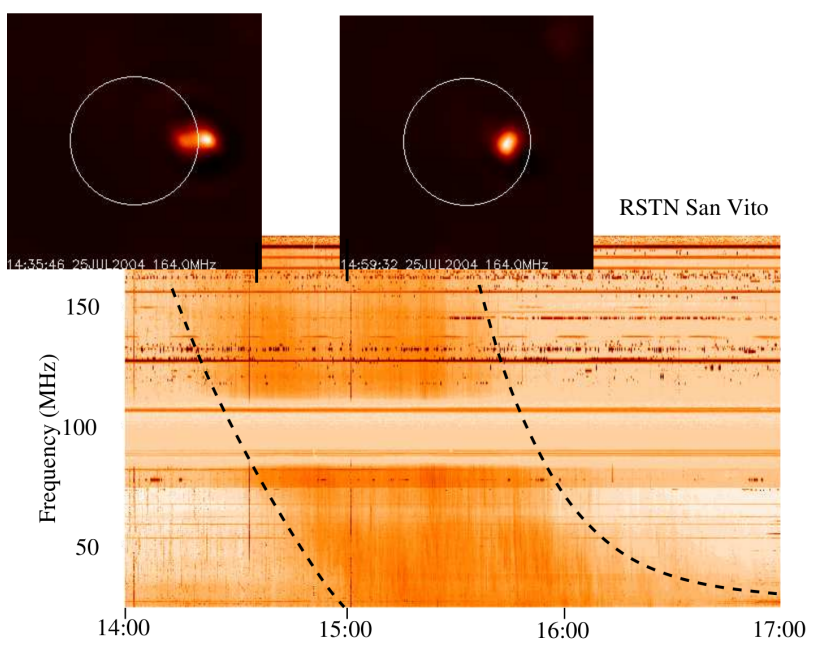

Figure 6: Metric type IV emission recorded by RSTN San Vito on 25 July 2004, in the frequency range of 180-25 MHz. Nancay Radioheliograph imaged the radio emission sources at $164 \mathrm{MHz}$, and those are shown at 14:35 UT and 14:59 UT. The type IV burst source was first located near the west limb and then moved toward the south-east, in between these images.

herent synchrotron emission from electrons with energies around $1 \mathrm{MeV}$.

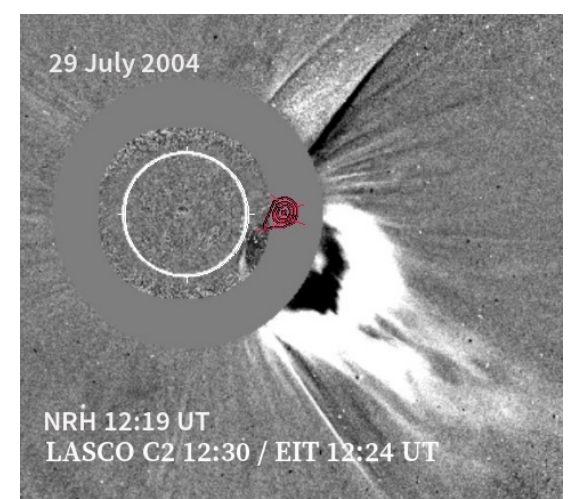

Figure 7: Nancay Radioheliograph (NRH) observation of the radio emission source at $164 \mathrm{MHz}$ at 12:19 UT on 29 July 2004 (red contours) plotted over the SOHO/LASCO C2/EIT difference image near that time. The metric type IV burst source at $164 \mathrm{MHz}$ is located over the west limb, seemingly near the north-western flank of the CME.

\section{Summary and discussion}

We have presented here observations of three different radio type IV burst events that occurred on 23,25 , and 29 July 2004, and originated from the same active region. The source location of the first event was near the disk center and in the third event near the west limb. Our analysis shows that in the last two events the type IV bursts experienced partial cut-offs in their emission, which coincided with the appearance of shock-related type II bursts.

In the 23 July 2004 event the short-duration type II burst was most probably caused by a shock at the flank of the first CME, or it was due to a bow shock near the leading front of the second CME. In a disk-centered event, a CME flank shock would not have been located in between the type IV burst and the observer, but for a bow shock at the CME front this would have been possible as the true CME heights could have been larger than the observed projected heights. In the 23 July event the IP type II burst ended near the time of the IP type IV burst appearance and the type IV burst emission was observed as a typical, uncut envelope structure.

The 25 July 2004 event consisted of two separate type II 


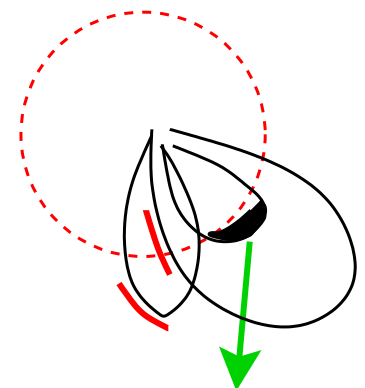

July 23: full type IV

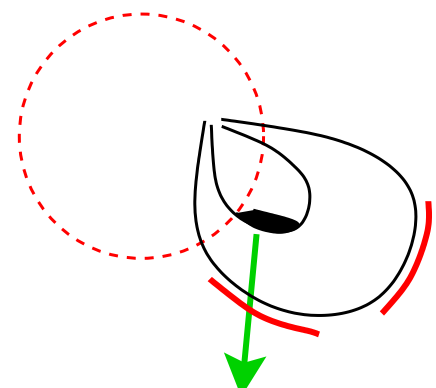

July 25: cut type IV

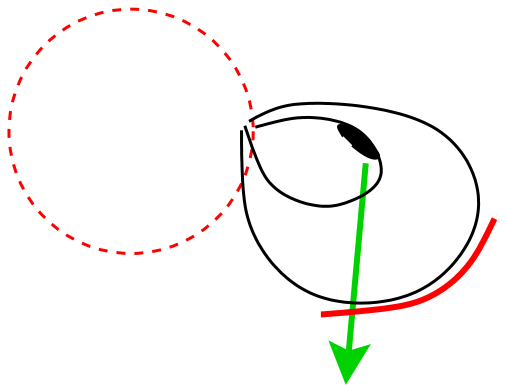

July 29: cut type IV

Figure 8: Schematic cartoons of the three events which show the suggested locations of the type II bursts (indicated by red lines) and the type IV emitting regions (filled black areas) inside the CME loops (not to scale, the radio emission sources could be several $\mathrm{R}_{\odot}$ from the Sun center). Green arrows show the direction toward the observer and where the type IV burst emission may cross the type II shock region.

bursts, of which the first, wide-band one was most likely formed by a CME bow shock since the estimated shock heights were well above the projected CME heights. The second, more narrow-band and slower shock that appeared in the radio spectrum two hours later was most probably due to a shock at the CME flanks, at relatively large heights. In principle, as two CMEs were observed to propagate in the same direction in the early stages of the event, the later shock could have been formed also inside the observed first CME. But, typically type II bursts that are associated with interacting and merging CMEs show fragmented and enhanced emission, as the densities in the wake of the first CME vary considerably. As this was not observed, the flank shock scenario looks to be more probable in this case. The partial disappearance of the IP type IV burst emission coincided with the appearance of this second, flank-shock related type II burst.

In the 29 July 2004 event the type IV burst also disappeared partially, near the time of the appearance of the wide-band type II burst, which was also determined to be due to a CME bow shock. In both events, on 25 and 29 July, the type II shock was most probably located in between the observer and the type IV burst source. Hence, the shock region could have absorbed or suppressed the type IV emission along this line of sight.

Based on these findings, we present a schematic cartoon in Figure 8, which shows the configurations in the three events. We propose that in the two events, on 25 and 29 July 2004, the type IV emission was partially blocked toward certain viewing angles by shock-related dense plasma.

The type IV bursts on 25 and 29 July were only weakly left-hand polarized (no polarization data was available for the 23 July event). This suggests that they were the moving type, non-directive, and emitted synchrotron radiation. The imaging of the type IV source region on 29 July also supports synchrotron as the emission mechanism. The source was located higher in the corona that would have been possible for a plasma emission source, within typical atmospheric densities.

Recently Vasanth et al. (2019) have presented analy- sis of a similar event, where the moving type IV emission source was also located very high in the corona, showed only weak polarization, but had too high brightness temperature to support the synchrotron radiation mechanism. Therefore Vasanth et al. (2019) suggested coherent plasma emission, excited by energetic electrons trapped within the top front of the bright core of a CME. They state, however, that further studies are needed to verify the emission mechanism.

The fine-structures inside the type IV bursts were similar in all our events, and they showed type III-like, narrowband fast-drift bursts within the frequency-drifting diffuse emission envelope. Unfortunately the time resolution in the radio spectral data was not high enough to verify if zebra patterns or similar were present. For example, frequency drifts with opposite directions within a single stripe have been observed earlier (Zlotnik et al., 2015), suggesting a nonuniform coronal magnetic trap.

\section{Conclusions}

We have presented here observations of three radio type IV burst events that occurred on three separate days but originated from the same active region. In the first event the source region was located near the solar disk center, in the second event near W30, and in the third event near the west limb. Our analysis shows that in the last two events the type IV bursts experienced partial cutoffs in their emission. These two bursts were estimated to be the moving type, i.e., associated with upward-moving CME structures instead of stationary coronal loops.

We conclude that partial cut-offs in IP type IV emission can appear also in moving type IV bursts, which are not supposed to be directive like stationary type IV bursts. The reason for the reduced emission could be absorption or suppression of emission toward certain viewing angles, caused by increased plasma density near CME flank and bow shock regions. High densities can be found especially in streamer regions, and streamers were observed near the CMEs in all three events. However, as the type II burst fre- 
quencies are much lower than those of the type IV bursts, they should not be able to block the radio propagation by means of reflection. As the type IV burst emission mechanism - or mechanisms - are not that well known either, this is clearly a topic that needs to be studied further.

If radio type IV burst emission can be observed only if the burst source is located near the solar disk center, this could be used to estimate the CME propagation direction. However, this is not possible if the observed directivity is caused by absorption/blocking of the type IV emission toward certain directions. Instead, the effect will tell if high density, shock-related regions are formed within CME structures, and where they may be located.

\section{Acknowledgements}

We thank all the individuals who have contributed in creating and updating the various solar event catalogues and databases. We are especially grateful to Stephen White for preparing and providing us the Green Bank Radio Burst Spectrometer data. We thank the RSDB service at LESIA/USN (Observatoire de Paris) for making the Nancay Radioheliograph data available. The CME catalog is generated and maintained at the CDAW Data Center by NASA and the Catholic University of America in cooperation with the Naval Research Laboratory.

\section{References}

Bastian, T.S., Pick, M., Kerdraon, A., Maia, D., Vourlidas, A., 2001. The Coronal Mass Ejection of 1998 April 20: Direct Imaging at Radio Wavelengths. Astrophys. J. 558, 1, L65-L69. https://doi.org/10.1086/323421

Boischot, A., 1957. Caractères d'un type d'émission hertzienne associé à certaines éruptions chromosphériques. Academie des Sciences Paris Comptes Rendus. 244, 1326-1329. http://adsabs.harvard.edu/abs/1957CRAS..244.1326B

Bougeret, J.-L., Goetz, K., Kaiser, M.L., et al., 2008. S/WAVES: The Radio and Plasma Wave Investigation on the STEREO Mission. Space Sci. Rev. 136, 487-528. https://doi.org/10.1007/s11214-0079298-8

Bougeret, J.-L., Kaiser, M.L., Kellogg, P.J., et al., 1995. WAVES: The radio and plasma wave investigation on the wind spacecraft. Space Sci. Rev. 71, 231-263. https://doi.org/10.1007/BF00751331

Bouratzis, C., Hillaris, A., Alissandrakis, C.E., et al., 2015. Fine Structure of Metric Type IV Radio Bursts Observed with the ARTEMIS-IV Radio-Spectrograph: Association with Flares and Coronal Mass Ejections. Solar Phys. 290, 219-286. https://doi.org/10.1007/s11207-014-0562-2

Bouratzis, C., Hillaris, A., Alissandrakis, C.E., et al., 2016. High resolution observations with Artemis-IV and the NRH. I. Type IV associated narrow-band bursts. Astron. Astrophys. 586, id. A29. https://doi.org/10.1051/0004-6361/201527229

Dulk, G.A., 1985. Radio emission from the sun and stars. Ann. Rev. Astron. Astrophys. 23, 169-224. https://doi.org/10.1146/annurev.aa.23.090185.001125

Gopalswamy, N., 1990. Clark Lake radio observations of coronal mass ejections. In Priest, E.R. and Krishan, V. (eds.), Basic plasma processes on the Sun, Proc. IAU Symp. No. 142, Kluwer Academic Publishers, Dordrecht, 495-500. http://adsabs.harvard.edu/abs/1990IAUS..142..495G

Gopalswamy, N., Akiyama, S., Mäkelä, P., Yashiro, S., Cairns, I.H., 2016. On the Directivity of Low-Frequency Type IV Radio Bursts. Presentation at the URSI Asia-Pacific Radio Science Conference in Seoul, August 21-25, 2016. https://arxiv.org/abs/1605.02223
Hillaris, A., Bouratzis, C., Nindos, A., 2016. Interplanetary Type IV Bursts. Solar Phys. 291, 2049-2069. https://doi.org/10.1007/s11207-016-0946-6

Kong, X., Chen, Y., Guo, F., et al., 2015. The Possible Role of Coronal Streamers as Magnetically Closed Structures in Shock-induced Energetic Electrons and Metric Type II Radio Bursts Astrophys. J. 798, id. 81. https://doi.org/10.1088/0004-637X/798/2/81

Kundu, M.R., 1965, Solar Radio Astronomy, Chapter 11: Type IV Radiation, Interscience Publishers, New York, 383-442.

Kundu, M.R., 1987. A type IV burst associated with a coronal streamer disruption event. Solar Phys. 111, 53-57. https://doi.org/10.1007/BF00145440

Liu, R., Lee, J., Wang, T., et al., 2010. A Reconnecting Current Sheet Imaged in a Solar Flare. Astrophys. J. Lett. 723, L28-L33. https://doi.org/10.1088/2041-8205/723/1/L28

Maia, D.J.F., Gama, R., Mercier, C., et al., 2007. The Radio-Coronal Mass Ejection Event on 2001 April 15. Astrophys. J. 660, 874-881. https://doi.org/10.1086/508011

Melnik, V.N., Rucker, H.O., Konovalenko, A.A., et al., 2008. Solar type IV bursts at frequencies 10-30 MHz. In Pingzhi Wang (ed.), Solar Physics Research Trends, Nova Science Publishers, Inc., 287-325.

Melnik, V.N., Brazhenko, A.I., Konovalenko, A.A., et al., 2018. Decameter Type IV Burst Associated with a Behind-the-limb CME Observed on 7 November 2013. Solar Phys. 293, id. 53. https://doi.org/10.1007/s11207-018-1271-z

Nindos, A., Aurass, H., Klein, K.-L., Trottet, G., 2008. Radio Emission of Flares and Coronal Mass Ejections. Invited Review. Solar Phys. 253, 3-41. https://doi.org/10.1007/s11207-008-9258-9

Pick, M., Vilmer, N., 2008. Sixty-five years of solar radioastronomy: flares, coronal mass ejections and Sun Earth connection. Astron. Astrophys. Rev. 16, 1-153. https://doi.org/10.1007/s00159-0080013-x

Pohjolainen, S., Allawi, H., Valtonen, E., 2013. Origin of wide-band IP type II bursts. Astron. Astrophys. 558, A7. https://doi.org/10.1051/0004-6361/201220688

Pohjolainen, S., van Driel-Gesztelyi, L., Culhane, J.L., Manoharan, P.K., Elliott, H.A., 2007. CME Propagation Characteristics from Radio Observations. Solar Phys. 244, 167-188. https://doi.org/10.1007/s11207-007-9006-6

Talebpour Sheshvan, N., Pohjolainen, S., 2018. Visibility and Origin of Compact Interplanetary Radio Type IV Bursts. Solar Phys. 293, id. 148. https://doi.org/10.1007/s11207-018-1371-9

Vasanth, V., Chen, Y., Lv, M., et al., 2019. Source Imaging of a Moving Type IV Solar Radio Burst and Its Role in Tracking Coronal Mass Ejection from the Inner to the Outer Corona. Astrophys. J. 870 , id. 30. https://doi.org/10.3847/1538-4357/aaeffd

Vrsnak, B., Magdalenic, J., Zlobec, P., 2004. Band-splitting of coronal and interplanetary type II bursts. III. Physical conditions in the upper corona and interplanetary space. Astron. Astrophys. 413, 753-763. https://doi.org/10.1051/0004-6361:20034060

Vrsnak, B., Zic, T., Falkenberg, T.V., et al., 2010. The role of aerodynamic drag in propagation of interplanetary coronal mass ejections. Astron. Astrophys. 512, id. A43. https://doi.org/10.1051/0004-6361/200913482

White, S.M., 2007. Solar Radio Bursts and Space Weather. Asian J. of Phys. 16, 189-207.

Zlotnik, E.Y., Zaitsev, V.V., Melnik, V.N., Konovalenko, A.A., Dorovskyy, V.V., 2015. 'Fingerprint' Fine Structure in the Solar Decametric Radio Spectrum Solar Physics. Solar Phys. 290, 2013-2030 https://doi.org/10.1007/s11207-015-0724-x 